\title{
Does the Peroneus Longus Tendon Autograft Affect the Ankle Function? A Case Series
}

\author{
Muhammad Hilman Bimadi, Krisna Yuarno Phatama, Edi Mustamsir
}

Department of Orthopaedic and Traumatology, Faculty of Medicine, Brawijaya University-Saiful Anwar General Hospital, Malang, Indonesia

\section{Abstract}

The peroneus longus (PL) tendon is one of the autograft choices for knee ligament injury because of its safety, length, and strength. However, studies that evaluated donor site morbidity after PL tendon graft are still limited. This case series aims to evaluate the functional outcomes of donor site morbidity after full-thickness PL tendon harvesting for knee ligament reconstruction.

This study evaluated four patients who had a revision of knee ligament reconstruction surgery using PL tendon autograft from August until September 2018. The functional outcome of the donor site was evaluated before surgery, two weeks, and three months after surgery by using functional scores assessment of foot and ankle.

There are some donor sites of autograft, but several studies show that the PL tendon autograft has proven to be more favorable methods for knee ligament reconstruction based on the accessibility, safety, and strength of the harvested tendon. The studies also found that using PL tendon as an autograft, has a good result in donor site morbidity evaluation compared to others. This study provides a satisfying result on the functional outcome of the donor site morbidity evaluation.

This study showed satisfactory functional outcomes of the foot and ankle after harvesting full-thickness PL tendon. All functional score assessment provides an excellent result in three months after surgery, although lateral ankle bulging has occurred in all patients. However, gait analysis and isokinetic test with a more significant number of patients and longer follow up periods are necessary for further study.

Keywords: Peroneus longus; Autograft; Donor site evaluation; Functional outcome; Foot and ankle

Level of Evidence: IV

\section{Article history}

Submitted: May 30th 2020

Revise : July $23^{\text {th }}, 2020$

Accepted : July $24^{\text {th }}, 2020$
Corresponding Author: Krisna Yuarno Phatama, MD, Department of Orthopaedic and Traumatology, Faculty of Medicine, Brawijaya University-Saiful Anwar General Hospital, Jalan Jaksa Agung Suprapto No. 2, Klojen, Malang, 65112, East Java, Indonesia Email of Krisna Yuarno Phatama: krisnayuarno@ub.ac.id 


\section{Introduction}

The second highest weight-bearing joint of the human body is a knee. There were ligamentous and involved cruciate ligaments which mainly provide knee stability ${ }^{1,2}$. According to the data, there were 238.488 knee ligament injuries occurred over the five years and often required for orthopaedic surgery ${ }^{3}$. Sports activity is the most common site for soft tissue injuries, particularly ligaments and tendons, obtained for more than $50 \%$ of all injuries. The patient morbidity appears from laceration, rupture, and inflammation of the tendon with disruption of quality of life ${ }^{4}$. The important thing for $\mathrm{ACL}$ reconstruction is to maintain knee stability and function that will allow returning to sport and avoid recurrent injury with many graft types, either autograft or allograft, which have already been studied widely ${ }^{1,4,5}$.

Bone-patellar-tendon-bone (BPTB) and hamstring autograft are the most commonly used procedure 5,6 . The advantages of BPTB may provide the greatest graft choice because it has a bone to bone healing, which consents the effective incorporation of tunnel and graft, and may allow a faster return to daily and sports activity ${ }^{5,7}$. However, the disadvantages is anterior knee pain, kneeling pain, loss of motion, patellar fracture, fat pad fibrosis, or patellar tendon contracture $^{5-7}$.

Furthermore, Hamstring autograft has another advantage, such as greater mechanical strength 1,5 , but it has been believed that it is difficult to predict the diameter of the harvested tendons before surgery, the potential decrease for hamstring power in the involved extremity, appearance of knee flexion strength deficiency, and could damage the saphenous nerve ${ }^{4-6}$. Subsequently, although the incidence of anterior knee pain and kneeling pain is lower than BPTB, it could occur, especially in Asians who frequently kneel as part of their daily activity ${ }^{7}$.

Therefore, many studies try to evaluate another reasonable autograft that would be useful in revision situations or as a complement to other autograft choices ${ }^{5-8}$. The choice of autograft that can be chosen as a new alternative is the peroneus longus (PL) tendon. Because of its length and strength, $\mathrm{PL}$ tendon was a suitable choice for $\mathrm{ACL}$ reconstruction, but there is little information that evaluated the donor site morbidity of PL tendon graft $1,4,8$. This study aims to evaluate the functional outcome of donor site morbidity after full-thickness PL tendon harvesting for knee ligament reconstruction by using the functional scores assessment system of foot and ankle.

\section{Case Presentation}

This study evaluated four patients from August until September 2018. All patients underwent revision of knee ligament reconstruction surgery using PL tendon autograft. All of the patients were male with a range of ages is 20-30 years. The surgery performed under general anesthesia. The patient positioned supine to simplify the access of the lateral malleolus for $\mathrm{PL}$ tendon graft. The longitudinal skin incision was made in the posterolateral side of the fibula, two centimeters above the lateral malleolus (Figure 1). At the posterior of the fibula, there was PL and peroneus brevis (PB) tendon. The brevis tendon is relatively flat, running directly posterior to the distal fibula while the PL tendon is more rounded

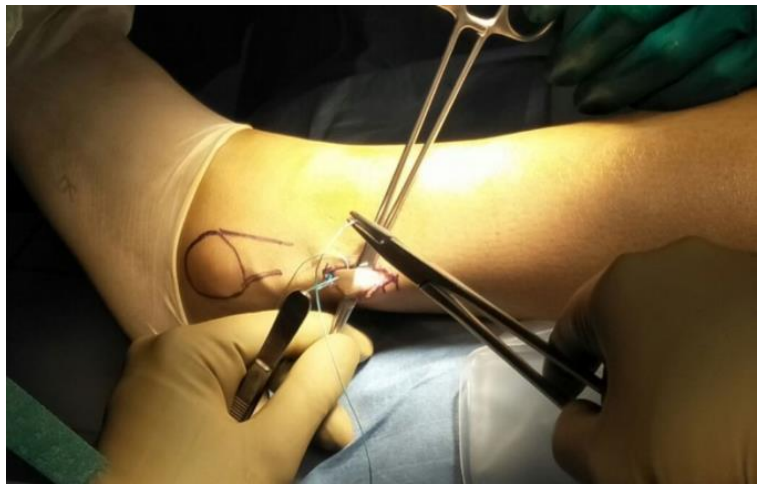

Figure 1. Clinical photograph of the PL-tendon during harvesting

and lies posterior to the brevis tendon. The distal end of the PL tendon was exposed with mosquito hemostat, sutured, and cutting off PL tendon at the distal end. Subsequently, the PL tendon was chosen to harvest by putting tendon stripper toward the proximal end and then pulling out the $\mathrm{PL}$ tendon. The superficial fascia and fat of the PL tendon were removed, and the rough edge was trimmed carefully. Furthermore, after PL tendon was completely harvested, the distal stump of PL 
tendon was sutured to the PB tendon. Therefore, the function of PL tendon still exists.

This study evaluates the functional outcome of the donor site after harvesting $\mathrm{PL}$ tendon for revision of knee ligament reconstruction in all the patients before surgery, two weeks and three months after surgery. This study uses functional score assessment for foot and ankle such as American Orthopaedic Foot and Ankle Society (AOFAS), Foot and Ankle Disability Index (FADI), St. Pierre, and Karlsson-Peterson scoring systems.

There was no finding for a decreased functional score of the foot and ankle from all the patients before the surgery (Table I). Nevertheless, the donor site was slightly bulging in all patients (Figure 2), and two weeks after surgery, the score was slightly decreased for all patients

\begin{tabular}{llllll} 
Table I. Foot and Ankle Functional Score & & & & \\
\hline & & FADI & AOFAS & ST. Pierre & $\begin{array}{l}\text { Karlsson- } \\
\text { Peterson }\end{array}$ \\
\hline Patient 1 & Before Surgery & 100 & 100 & 0 & 100 \\
ACL Reconstruction & Two Weeks After Surgery & 95 & 96 & 4 & 95 \\
& Three Months After Surgery & 100 & 100 & 0 & 100 \\
\hline Patient 2 & Before Surgery & 100 & 100 & 0 & 100 \\
PLC Reconstruction & Two Weeks After Surgery & 94 & 94 & 3 & 96 \\
& Three Months After Surgery & 100 & 100 & 0 & 100 \\
\hline Patient 3 & Before Surgery & 100 & 100 & 0 & 100 \\
ACL Reconstruction & Two Weeks After Surgery & 97 & 96 & 3 & 96 \\
& Three Months After Surgery & 100 & 100 & 0 & 100 \\
\hline Patient 4 & Before Surgery & 100 & 100 & 0 & 100 \\
ACL Reconstruction & Two Weeks After Surgery & 95 & 98 & 3 & 95 \\
& Three Months After Surgery & 100 & 100 & 0 & 100 \\
\hline
\end{tabular}

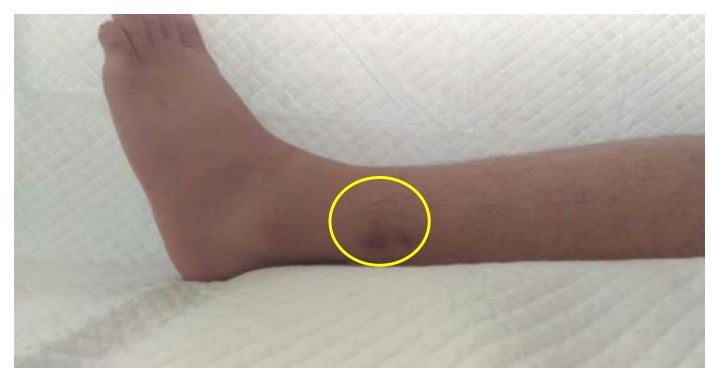

Figure 2. Clinical photograph showed that the donor site was slightly bulging after surgery

without significant foot and ankle problems. Furthermore, the function of the foot and ankle returned to normal in three months after surgery which is shown by all score criteria.

\section{Discussion}

There are limited studies that highlight donor site morbidity of the ankle in patients who underwent $\mathrm{PL}$ tendon harvesting for knee ligament reconstruction ${ }^{9}$. According to the high incidence of knee ligament injury, evaluation of donor site morbidity after harvesting the graft needs to be considered, especially for PL tendon autograft, which increases to be a more favourable choice of autograft for knee ligament reconstruction ${ }^{10,11}$. Each of the autograft sources has a unique characteristic. However, the previous study showed that there was a better functional outcome in knee ligament reconstruction using the $\mathrm{PL}$ tendon compared with the hamstring tendon ${ }^{10}$. Rudy et al. ${ }^{4}$ reported that the mean value of tensile strength test on 12 samples between peroneus tendon and hamstring tendon was $446.16 \mathrm{~N}$ and $405.88 \mathrm{~N}$. The study showed that even though the average of tensile strength in peroneus tendon is slightly higher, but statistically the tensile strength of both tendons are nearly the same ${ }^{4}$.

Graft selection plays an essential role in achieving satisfactory clinical outcomes in anatomic reconstruction because there are advantages and disadvantages of each graft and harvesting procedures ${ }^{8}$. The requirement of tendon structure to become an ideal autograft is to have an acceptable amount of strength, should be easily and safely harvested, and when it was taken from the donor site, the process should not result in any apparent functional impairment ${ }^{5-8}$. Donor site morbidity after tendon harvest procedure can be categorized as a structural failure and functional impairment ${ }^{8}$. Furthermore, the PL tendon has an essential role in plantar flexion of the ankle, eversion of the foot, maintenance of the transverse arch, and steadying of the leg over the foot ${ }^{8-10}$. The PL tendon has a synergistic mechanism with the PB tendon. For stability, the PL tendon works in combination with the $\mathrm{PB}$ tendon to distribute pressure on the forefoot ${ }^{10}$. The strength levels on the PL and PB tendons are equal, and the study revealed that there was no difference in ankle function if the PL tendon was harvested ${ }^{10}$.

In contrast, the previous study reported that there is a deterioration of donor site function 
after harvesting PL tendon, including decreased peak torque eversion and inversion, decreased ankle function, and ankle instability. The clinical evaluations of mean follow up using varus talar tilt tests found laxity in $8.4 \%$ of all the patients, and decreased power of eversion and first ray plantarflexion in $16.7 \%$ of all the patients. Only one patient with ankle instability was found in the first six months after the surgery. In addition, the study performed isokinetic test at seven months means to follow up and found that there was significant deterioration of eversion and invention at both velocities $(60 \%$ second and $120 \%$ second). These findings revealed that the ankle instability might occur on a patient who lost PL tendon in the first 12 months after surgery. However, there was no significant impairment of ankle function after harvesting full-thickness $\mathrm{PL}$ tendon by the evaluations of ankle scores as AOFAS and VAS-FA score ${ }^{9}$. Subsequently, on the other hand, it found a similar result with this study through the evaluation of ankle score. The function of the donor site was excellent after harvesting PL tendon according to functional ankle test based on FADI and AOFAS score. This probably because while the PL was harvested, the PB is still intact, where the previous studies mentioned $\mathrm{PB}$ to be more effective evertor of the ankle and will maintain ankle eversion function after the harvest of PL tendon ${ }^{12-15}$. Moreover, the anterior half of the peroneus longus tendon (AHPLT) was also reported to be an acceptable alternative for autograft use concerning its strength, safety, and donor site morbidity ${ }^{12,16}$.

There was no significant impairment of the foot and ankle function after the harvest of the AHPLT based on pre and postoperative AOFAS and FADI scoring systems obtained from all patients, including the study who underwent a variety of knee ligament reconstructions with the AHPLT graft and followed up for two years. Also, no signs of $\mathrm{PL}$ tendon rupture, peroneal nerve injury, tendinopathy, or difference in eversion and first ray plantarflexion muscle strength between the donor site and contralateral healthy site ${ }^{10,12}$. In line with the statement, this study shows that the functional outcome of the donor site after harvesting the full thickness PL tendon for knee ligament injury provides an excellent result. There was no significant impairment of foot and ankle function before surgery, and three months after surgery by using functional scores assessment such as FADI, AOFAS, St. Pierre, and KarlssonPeterson scoring systems. Although the functional score of the ankle slightly decreased on two weeks after surgery, the patient's experience shows that all problems and functions of the ankle returned to normal in three months after surgery, based on all of the scoring systems.

Despite all the positive results, this study also has some limitations. The main concern is the followup period which is too short. Therefore, ankle instability, which may appear latter, cannot be examined. Another concern is the limited tool used to analyze ankle functional status which are only AOFAS, FADI, St. Pierre, and KarlssonPeterson scoring systems. The use of isokinetic test and gait analysis is also suggested to evaluate the function of the ankle for further studies. Moreover, this study also lacks the number of subjects. Thus, further study with a bigger sample is suggested. Last but not least, this study lacks the number of images representing every subject hence the limitation in showing the patient's condition. However, this present study showed results, which may be valuable information for future studies.

\section{Conclusions}

The full-thickness PL tendon autograft has no foot and ankle functional effect shown by all scoring systems for three months follow up despite the occurrence of lateral ankle bulging in all patients. However, this study is limited by the short follow up period, number of tools, number of subjects, and number of figures. Thus, future studies are necessary to evaluate gait analysis and isokinetic test with a more significant number of patients and longer follow up periods.

\section{Conflicts of interest}

The authors declared that there is no conflict of interest regarding this study.

\section{Acknowledgements}

This research paper is made possible by God's grace and through the support from parents, family, and friends. 


\section{References}

1. Barzegar M, Hosseini A, Karimi M, Nazem K. Can we use peroneus longus in addition to hamstring tendons for anterior cruciate ligament reconstruction? Adv Biomed Res. 2014;3(1):115.

2. Song $X, L i Q, W u Z, X u Q$, Chen $D$, Jiang $Q$. Predicting the graft diameter of the peroneus longus tendon for anterior cruciate ligament reconstruction. Med (United States). 2018;97(44).

3. Gianotti SM, Marshall SW, Hume PA, Bunt L. Incidence of anterior cruciate ligament injury and other knee ligament injuries: $A$ national population-based study. J Sci Med Sport. 2009;12(6):622-627.

4. Rudy, Mustamsir E, Phatama KY. Tensile strength comparison between peroneus longus and hamstring tendons: A biomechanical study. Int J Surg Open. 2017;9:41-44.

5. Rhatomy $S$, Asikin AIZ, Wardani $A E$, Rukmoyo T, Lumban-Gaol I, Budhiparama NC. Peroneus longus autograft can be recommended as a superior graft to hamstring tendon in single-bundle $\mathrm{ACL}$ reconstruction. Knee Surgery, Sport Traumatol Arthrosc. 2019;27(11):35523559.

6. Shi FD, Hess DE, Zuo JZ, et al. Peroneus Longus Tendon Autograft is a Safe and Effective Alternative for Anterior Cruciate Ligament Reconstruction. J Knee Surg. 2019;32(8):804-811.

7. Setyawan R, Soekarno NR, Asikin AIZ, Rhatomy S. Posterior Cruciate Ligament reconstruction with peroneus longus tendon graft: 2-Years follow-up. Ann Med Surg. 2019;43(March):38-43.

8. Park CH, Lee WC. Donor Site Morbidity after Lateral Ankle Ligament Reconstruction Using the Anterior Half of the Peroneus Longus Tendon Autograft. Am J Sports Med. 2017;45(4):922-928.

9. Chayanin A, Chernchujit B, A. A, K. C, P. N, K. $\mathrm{S}-\mathrm{I}$. The anterior cruciate ligament reconstruction with the peroneus longus tendon: A biomechanical and clinical evaluation of the donor ankle morbidity. $J$
Med Assoc Thail. 2015;98(6):555-560.

10. Rhatomy S, Wicaksono FH, Soekarno NR, Setyawan R, Primasara S, Budhiparama NC. Eversion and First Ray Plantarflexion Muscle Strength in Anterior Cruciate Ligament Reconstruction Using a Peroneus Longus Tendon Graft. Orthop J Sport Med. 2019;7(9):1-5.

11. Sasetyo DR, Rhatomy S, Pontoh LA. Peroneus longus tendon: The promising graft for anterior cruciate ligament reconstruction surgery. Asia-Pacific J Sport Med Arthrosc Rehabil Technol. 2017;9(2017):25.

12. Kim HN, Jeon JY, Dong Q, et al. Lateral ankle ligament reconstruction using the anterior half of the peroneus longus tendon. Knee Surgery, Sport Traumatol Arthrosc. 2015;23(6):1877-1885.

13. Rhatomy S, Hartoko L, Setyawan R, et al. Single bundle $A C L$ reconstruction with peroneus longus tendon graft: 2-years follow-up. J Clin Orthop Trauma. 2019;(xxxx):6-10.

14. Davda K, Malhotra K, O'Donnell P, Singh D, Cullen N. Peroneal tendon disorders. EFORT Open Rev. 2017;2(6):281-292.

15. Otis JC, Deland JT, Lee S, Gordon J. Peroneus brevis is a more effective evertor than peroneus longus. Foot Ankle Int. 2004;25(4):242-246.

16. Sun $\mathrm{Y}$, Wang $\mathrm{H}$, Tang $\mathrm{Y}$, Zhao $\mathrm{H}$, Qin S, Zhang F. Reconstruction of the lateral ankle ligaments using the anterior half of peroneus longus tendon graft. Foot Ankle Surg. 2019;25(2):242-246. 